To be published in ApJ, March 20, 2000 (Vol.532)

\title{
Tidal Interaction between a Fluid Star and a Kerr Black Hole in Circular Orbit
}

\author{
Paul Wiggins ${ }^{1}$ and Dong Lai \\ Center for Radiophysics and Space Research, Department of Astronomy, Cornell University, \\ Ithaca, NY 14853 \\ E-mail: wiggins,dong@spacenet.tn.cornell.edu
}

\begin{abstract}
We present a semi-analytic study of the equilibrium models of close binary systems containing a Newtonian fluid star (mass $m$ and radius $R_{0}$ ) and a Kerr black hole (mass $M)$ in circular orbit. We consider the limit $M \gg m$ where spacetime is described by the Kerr metric. The tidally deformed star is approximated by an ellipsoid, and satisfies the polytropic equation of state. The models also include fluid motion in the stellar interior, allowing binary models with nonsynchronized stellar spin (as expected for coalescing neutron star-black hole binaries) to be constructed. Our relativistic, compressible Roche-Riemann model is a generalization of the incompressible, corotating Roche model studied earlier by Fishbone (1973).

Tidal disruption occurs at orbital radius $r_{\text {tide }} \sim R_{0}(M / m)^{1 / 3}$, but the dimensionless ratio $\hat{r}_{\text {tide }}=r_{\text {tide }} /\left[R_{0}(M / m)^{1 / 3}\right]$ depends on the spin parameter of the black hole as well as on the equation of state and the internal rotation of the star. We find that the general relativistic tidal field disrupts the star at a larger $\hat{r}_{\text {tide }}$ than the Newtonian tide; the difference is particularly prominent if the disruption occurs in the vicinity of the black hole's horizon. In general, $\hat{r}_{\text {tide }}$ is smaller for a (prograde rotating) Kerr black hole than for a Schwarzschild black hole.

We apply our results to coalescing black hole-neutron star and black hole-white dwarf binaries. The tidal disruption limit is important for characterizing the expected gravitational wave signals and is relevant for determining the energetics of gamma ray bursts which may result from such disruption.
\end{abstract}

Subject headings: stars: neutron - stars: black hole - stars: white dwarf - gravitation - hydrodynamics - gamma ray burst

\footnotetext{
${ }^{1}$ Also Department of Applied and Engineering Physics, Cornell University.
} 


\section{Introduction}

The problem of tidal disruption of fluid stars by black holes in close binaries was first studied in the 1970s (e.g., Fishbone 1973; Mashhoon 1975). Lattimer \& Schramm (1976) specifically considered neutron star disruption, and focused on mass ejection in such an event and its implications for nucleosynthesis. In recent years, coalescing binary systems containing a black hole (BH) and a neutron star (NS) or a white dwarf (WD) have become increasingly important both because they are one of the most promising sources of gravitational waves (GW) to be detected by ground-based laser interferometric GW detectors (LIGO/VIRGO/TAMA/GEO) or spaceborne interferometers such as LISA (see Thorne 1997 for a review), and because they potentially serve as a central engine for gamma ray bursts (e.g., Narayan, Paczynski \& Piran 1992; Meszaros, Rees \& Wijers 1998; Piran 1998).

Current numerical simulations of NS-BH binaries and WD-BH binaries (Kluzniak \& Lee 1998; Fryer et al. 1998; Uryu \& Eriguchi 1999; Janka et al. 1999) are based on Newtonian theory (in some cases, the gravitational field of the $\mathrm{BH}$ is approximated by a pseudo-Newtonian potential), and mostly (with the exception of Janka et al. 1999) neglect gravitational radiation reaction on the dynamics. While such simulations provide useful insight, full understanding of the coalescence will have to wait for the development of numerical relativity.

In this paper, we develop a semi-analytic model of close binaries containing a fluid star (mass $m$ ) and a massive $\mathrm{BH}$ (mass $M$ ). To make the problem tractable, we make a series of approximations. The obvious simplification is that in the limit $M \gg m$, the spacetime can be approximated by the Kerr metric. Furthermore, we assume that the radius of the companion star, $R_{0}$, is much greater than $G m / c^{2}$, allowing the self gravity to be treated in Newtonian theory. The companion star is modeled using the standard ellipsoid ansatz (Chandrasekhar 1969) and its compressible generalization (Lai, Rasio \& Shapiro 1993,1994; hereafter LRS1 and LRS2). This has the advantage that it reduces the infinite degrees freedom and the partial differential equations to a set of coupled ordinary differential equations. These equations are then reduced to coupled algebraic equations by demanding quasi-equilibrium. Fishbone (1973) has constructed "relativistic Roche models" where the incompressible fluid body is tidally locked with the orbital motion. However, it is necessary to consider models where internal fluid motion is allowed, since in a coalescing binary the NS spin is not expected to be synchronized with the orbit (Kochanek 1992; Bildsten \& Cutler 1992). Newtonian Roche-Riemann binary model has been studied in LRS1. The "relativistic Roche-Riemann model" (the subject of this paper) represents a generalization to these previous works by allowing for nonsynchronized stellar spin and including general relativistic tidal field. We note that Shibata (1996) has studied some aspects of the relativistic Roche-Riemann model where the fluid body

is assumed incompressible. Post-Newtonian correction in binary ellipsoid models has also been studied (e.g., Taniguchi \& Shibata 1997; Shibata \& Taniguchi 1997; Taniguchi 1999).

Our relativistic Roche-Riemann models (and other semi-analytic models based on ellipsoid) clearly have limitations (see also $\S 7$ ). For example, at small orbital radii, higher-order tidal fields 
are expected to be important and the star will not have an exact ellipsoid shape. In the case of NS-BH binaries, the conditions $M / m \gg 1$ and $R_{0} c^{2} /(G m) \gg 1$ are satisfied only approximately. However, given that fully relativistic numerical calculations are still prohibitively difficult, our analytic models have the virtue in that they provide a wide array of semi-quantitative information about the behavior of the these systems. For example, some of the questions we address in this paper are: How does the tidal limit in general relativity compare with that in Newtonian gravity? How does the tidal limit depend on the spin parameter of the $\mathrm{BH}$ (and other parameters related to the NS equation of state and internal rotation)? The answers to these questions are important for determining the gravitational wave signals and the energetics of the gamma ray bursts that may result from the tidal disruption. While the absolute value of the tidal limit calculated from our model may not be accurate, its dependence on various parameters should be robust (see $\S 7$ ), and can be used to calibrate results from numerical simulations when they become available.

In $\S 2$ and $\S 3$ we present the basic equations of our model and derive the scaling relations. Numerical results are given in $\S 4$, where we focus on the tidal limits for different models. The tidal effect on the orbital dynamics is considered in $\S 5$, and in $\S 6$ we discuss possible applications of our results to gamma ray bursts. In $\S 7$ we comment on the validity and limitations of our models. Throughout the paper we shall use units such that $G=c=1$.

\section{Basic Equations}

\subsection{Tidal tensor}

The formalism of calculating tidal tensor in general relativity has been studied by many authors (e.g., Fishbone 1973; Mashhoon 1975; Marck 1983). The basic calculations and results are briefly summarized as follows. Consider a body (with mass $m$ much smaller than the BH mass $M$ ) on a geodesic in the Kerr metric. (i) One starts with the equation of geodesic deviation:

$$
\frac{d^{2} n^{\alpha}}{d \tau^{2}}+R_{\beta \gamma \delta}^{\alpha} u^{\beta} n^{\gamma} u^{\delta}=0
$$

where $\mathbf{u}$ is the 4 -velocity of the center-of-mass, $\mathbf{n}$ is the 4 -separation vector, $R_{\beta \gamma \delta}^{\alpha}$ is the Riemann tensor, which can be calculated in certain convenient basis; (ii) Construct a parallel-transporting orthonormal basis carried by the body: $\left\{\mathbf{e}_{(0)}, \mathbf{e}_{(1)}, \mathbf{e}_{(2)}, \mathbf{e}_{(3)}\right\}$, with $\mathbf{e}_{(0)}=\mathbf{u}$, and satisfies the parallel transport equation $\nabla_{\mathbf{u}} \mathbf{e}_{(a)}=0(a=0,1,2,3)$; (iii) The geodetic deviation equation can be reduced to

$$
\frac{d^{2} X_{i}}{d \tau^{2}}+\sum_{j} C_{i j} X_{j}=0, \quad(i=1,2,3),
$$

where $X_{i} \equiv \mathbf{e}_{(i) \mu} n^{\mu}$, and the tidal tensor is

$$
C_{i j}=R_{\alpha \beta \gamma \delta} \mathbf{e}_{(0)}^{\alpha} \mathbf{e}_{(i)}^{\beta} \mathbf{e}_{(0)}^{\gamma} \mathbf{e}_{(j)}^{\delta} .
$$


We set up the parallel-transporting frame as in Figure 1, with $\mathbf{e}_{(3)}$ perpendicular to the orbital plane. The nonzero components of the tidal tensor in this frame is given by (Marck 1983)

$$
\begin{aligned}
C_{11} & =\frac{M}{r^{3}}\left(1-3 \frac{r^{2}+K}{r^{2}} \cos ^{2} \Psi\right), \\
C_{22} & =\frac{M}{r^{3}}\left(1-3 \frac{r^{2}+K}{r^{2}} \sin ^{2} \Psi\right), \\
C_{33} & =\frac{M}{r^{3}}\left(1+3 \frac{K}{r^{2}}\right), \\
C_{12}=C_{21} & =\frac{M}{r^{3}}\left(-\frac{3}{2} \frac{r^{2}+K}{r^{2}} \sin 2 \Psi\right) .
\end{aligned}
$$

Here the angle $\Psi$ is specified by the equation

$$
\dot{\Psi}=\frac{d \Psi}{d \tau}=\frac{E\left(L_{z}-a E\right)+a}{r^{2}+K},
$$

where $E$ is the the energy (per unit mass), $L_{z}$ is the $z$-orbital angular momentum (per unit mass), $K=\left(L_{z}-a E\right)^{2}$ is the Carter constant (with $Q=0$ ), and the $\operatorname{dot}$ (".") denotes $d / d \tau$ in this and all the following equations. In circular orbit, we have (e.g., Misner, Thorne, \& Wheeler 1973)

$$
\begin{aligned}
E & =\frac{r^{2}-2 M r+a \sqrt{M r}}{r \sqrt{P}}, \\
L_{z} & =\frac{\sqrt{M r}\left(r^{2}-2 a \sqrt{M r}+a^{2}\right)}{r \sqrt{P}},
\end{aligned}
$$

where $P=r^{2}-3 M r+2 a \sqrt{M r}$. Thus we have

$$
K=\left(a E-L_{z}\right)^{2}, \quad \dot{\Psi}=\left(\frac{M}{r^{3}}\right)^{1 / 2} .
$$

Note that in general relativity, the angle $\Psi$ is not equal to $\theta$, the true anomaly of the orbit, which satisfies

$$
\dot{\theta}=\left[\frac{2 M}{r} a E+\left(1-\frac{2 M}{r}\right) L_{z}\right]\left(r^{2}+a^{2}-2 M r\right)^{-1}
$$

\subsection{Fluid Equations}

Including internal pressure and self-gravity, the Euler equation in the parallel-transporting frame reads

$$
\frac{d V_{i}}{d \tau}=-\frac{1}{\rho} \frac{\partial P}{\partial X_{i}}-\frac{\partial \Phi}{\partial X_{i}}-\sum_{j} C_{i j} X_{j}
$$

where $V_{i}=d X_{i} / d \tau$ and $\Phi$ is the potential for the (Newtonian) self-gravity. It is convenient to set up another frame, tied to the principal axis of the body (see Fig. 1). This "principal frame" is 
related to the parallel-transporting frame by a rotation of angle $\phi$ :

$$
x_{i}=\sum_{j} T_{i j} X_{j}
$$

where $T_{11}=T_{22}=\cos \phi, T_{12}=-T_{21}=\sin \phi, T_{33}=1$, and the other elements are zero. In the principal frame, the Euler equation becomes:

$$
\ddot{\mathbf{x}}+2 \boldsymbol{\Omega} \times \dot{\mathbf{x}}+\boldsymbol{\Omega} \times(\boldsymbol{\Omega} \times \mathbf{x})+\dot{\boldsymbol{\Omega}} \times \mathbf{x}=-\frac{1}{\rho} \nabla P-\nabla \Phi-\nabla \Phi_{\text {tide }},
$$

where

$$
\Phi_{\text {tide }}=\frac{1}{2} \sum_{i j} c_{i j} x_{i} x_{j} .
$$

The tidal tensor in the principal frame is

$$
c_{i j}=\sum_{k l} T_{i k} T_{j l} C_{k l}
$$

The nonzero components are:

$$
\begin{aligned}
c_{11} & =\frac{M}{r^{3}}\left[1-3 \frac{r^{2}+K}{r^{2}} \cos ^{2}(\Psi-\phi)\right], \\
c_{22} & =\frac{M}{r^{3}}\left[1-3 \frac{r^{2}+K}{r^{2}} \sin ^{2}(\Psi-\phi)\right], \\
c_{33} & =\frac{M}{r^{3}}\left(1+3 \frac{K}{r^{2}}\right), \\
c_{12}=c_{21} & =\frac{M}{r^{3}}\left[-\frac{3}{2} \frac{r^{2}+K}{r^{2}} \sin 2(\Psi-\phi)\right] .
\end{aligned}
$$

\subsection{Fluid Equations in the Ellipsoid Representation}

Since the tidal force is a linear function of $x_{i}$, for an incompressible fluid, ellipsoidal figures describe exactly the solution of the fluid equation (15) (Chandrasekhar 1969). We can further generalize the ellipsoid figures to compressible fluid by assuming that the surfaces of constant density inside the star form self-similar ellipsoids and that the the velocity of a fluid element is a linear function of $x_{i}$ (LRS1,LRS2). These approximations reduce the infinite degrees of freedom and partial differential equations, governing the dynamics of the companion, to five dynamic variables associated with the body and a set of ordinary differential equations. The five dynamic variables are the three principal axes $a_{1}, a_{2}, a_{3}$ and two angles $\phi, \lambda$, defined such that $d \phi / d \tau=\Omega$ and $d \lambda / d \tau=\Lambda$. Here $\Omega$ is the angular velocity of the ellipsoidal figure measured in the parallel transporting frame, and $\Lambda$ characterizes the internal fluid motion (see eq. [24] below) via $\Lambda=-a_{1} a_{2} \zeta /\left(a_{1}^{2}+a_{2}^{2}\right)$ with $\zeta$ the (uniform) vorticity along the $z$-axis in the frame corotating with the figure. The physical 
significance of $\phi$ is illustrated in Fig. 1, but $\lambda$ is without meaning and only its derivative, $\Lambda$, appears in our equations. The orbit is specified by the orbital separation $r$ and the true anomaly $\theta$.

Several parameters must be given to complete the description of the system: In addition to the stellar mass $m$, the $\mathrm{BH}$ mass $M$ (satisfying $M \gg m$ ) and the Kerr parameter $a$, there are parameters associated with the equation of state and the initial stellar rotation. We adopt the polytropic equation of state,

$$
P=K \rho^{1+1 / n},
$$

with $n$ the polytropic index. Note that $K$ (not to be confused with that $K$ in the tidal tensor; see eqs. [4]-[7]) is uniquely determined from $n, m$ and $R_{0}$, the radius of the nonrotating star with the same mass. Thus instead of $K$, we shall use $R_{0}$ as a parameter. Finally, we allow the star to have some initial rotation, $\Omega_{s}$, when the binary separation is infinite (see $\S 4.3$ ).

The equations governing the dynamics of the system can be obtained using the variational principle. It is convenient to write the Lagrangian as the sum of two terms:

$$
L=L_{\text {body }}+L_{\text {tide }}
$$

We assume that the star moves on a circular geodesic around the $\mathrm{BH}$, and neglect tidal effect on the orbital motion - This is an excellent approximation for $M \gg m$ even if the star is highly distorted (see $§ 5)$. Thus the orbital terms do not appear in the Lagrangian. The internal Lagrangian of the body, $L_{\text {body }}$, is exactly the same as in the Newtonian treatment. The velocity field $\mathbf{u}$ of the fluid inside the star relative to its center of mass can be written as

$$
\mathbf{u}=\left[\left(\frac{a_{1}}{a_{2}} \Lambda-\Omega\right) x_{2} \mathbf{e}_{1}+\left(-\frac{a_{2}}{a_{1}} \Lambda+\Omega\right) x_{1} \mathbf{e}_{2}\right]+\left(\frac{\dot{a}_{1}}{a_{1}} x_{1} \mathbf{e}_{1}+\frac{\dot{a}_{2}}{a_{2}} x_{2} \mathbf{e}_{2}+\frac{\dot{a}_{3}}{a_{3}} x_{3} \mathbf{e}_{3}\right),
$$

where $\mathbf{e}_{1}, \mathbf{e}_{2}$ and $\mathbf{e}_{3}$ are the basis unit vectors along the principal axes of the ellipsoid. On integration, the kinetic energy of the star relative to its center of mass is given by

$$
T=\frac{1}{2} I\left(\Lambda^{2}+\Omega^{2}\right)-\frac{2}{5} \kappa_{n} m a_{1} a_{2} \Lambda \Omega+\frac{1}{10} \kappa_{n} m\left(\dot{a}_{1}^{2}+\dot{a}_{2}^{2}+\dot{a}_{3}^{2}\right)
$$

with $I=\kappa_{n} m\left(a_{1}^{2}+a_{2}^{2}\right) / 5$ and where $\kappa_{n}$ is a constant depending on $n$ and specifies the mass concentration inside the star (see Table 1 in LRS1). The internal energy is:

$$
U=\int d m n \frac{P}{\rho}=k_{1} K \rho_{c}^{1 / n} m
$$

where $k_{1}$ is another constant depending only on $n$, and $\rho_{c} \propto m /\left(a_{1} a_{2} a_{3}\right)$ is the central density. The self gravitational potential energy is given by:

$$
W=-\frac{3}{5-n} \frac{m^{2}}{R} \frac{\mathcal{I}}{2 R^{2}}, \text { with } \mathcal{I}=A_{1} a_{1}^{2}+A_{2} a_{2}^{2}+A_{3} a_{3}^{2},
$$

where $R \equiv\left(a_{1} a_{2} a_{3}\right)^{1 / 3}$ is the mean radius of the ellipsoid, and the dimensionless index symbols $A_{i}$ are defined as in Chandrasekhar $(1969 ; \S 17)$. The Lagrangian for the star is then

$$
L_{\text {body }}=T-U-W .
$$


Using the tidal tensor derived earlier (see eq. [16]), the tidal Lagrangian may be calculated as

$$
L_{\text {tide }}=-W_{\text {tide }}=-\int d^{3} x \rho(\mathbf{x}) \Phi_{\text {tide }}(\mathbf{x})=-\frac{1}{2}\left(I_{11} c_{11}+I_{22} c_{22}+I_{33} c_{33}\right),
$$

with $I_{i j}=\kappa_{n} m a_{i}^{2} \delta_{i j} / 5$.

The dynamic equations of the system are found using the Euler-Lagrange equation:

$$
\frac{d}{d \tau}\left(\frac{\partial L}{\partial \dot{q}_{i}}\right)=\frac{\partial L}{\partial q_{i}}
$$

where $q_{i}$ represents one of the dynamic variables $\left\{a_{1}, a_{2}, a_{3}, \phi, \lambda\right\}$. We find

$$
\begin{aligned}
\ddot{a}_{1} & =a_{1}\left(\Omega^{2}+\Lambda^{2}\right)-2 a_{2} \Omega \Lambda-\frac{2 \pi}{q_{n}} a_{1} A_{1} \bar{\rho}+\frac{5 k_{1} P_{c}}{n \kappa_{n} \rho_{c} a_{1}}-c_{11} a_{1}, \\
\ddot{a}_{2} & =a_{2}\left(\Omega^{2}+\Lambda^{2}\right)-2 a_{1} \Omega \Lambda-\frac{2 \pi}{q_{n}} a_{2} A_{2} \bar{\rho}+\frac{5 k_{1} P_{c}}{n \kappa_{n} \rho_{c} a_{2}}-c_{22} a_{2}, \\
\ddot{a}_{3} & =-\frac{2 \pi}{q_{n}} a_{3} A_{3} \bar{\rho}+\frac{5 k_{1} P_{c}}{n \kappa_{n} \rho_{c} a_{3}}-c_{33} a_{3}, \\
\dot{J}_{s} & =\frac{3}{10} \kappa_{n} m \frac{M}{r^{3}} \frac{r^{2}+K}{r^{2}}\left(a_{1}^{2}-a_{2}^{2}\right) \sin [2(\Psi-\phi)], \\
\dot{\mathcal{C}} & =0,
\end{aligned}
$$

where we have defined

$$
\begin{aligned}
J_{s} & \equiv I \Omega-\frac{2}{5} \kappa_{n} m a_{1} a_{2} \Lambda \\
\mathcal{C} & \equiv I \Lambda-\frac{2}{5} \kappa_{n} m a_{1} a_{2} \Omega
\end{aligned}
$$

and $\bar{\rho} \equiv 3 m /\left(4 \pi R^{3}\right), q_{n} \equiv \kappa_{n}(1-n / 5)$. The quantity $J_{s}$ is the spin angular momentum of the star and $\mathcal{C}$ is interpreted as the circulation in the locally nonrotating inertial frame. In the absence of viscosity, Kelvin's theorem ensures that the circulation is conserved. Note that the corotation case corresponds to $\Lambda=0$.

For a binary system in quasi-equilibrium, we require

$$
\ddot{a}_{i}=0, \phi=\Psi, \text { and } \dot{\phi}=\Omega=\dot{\Psi}=\sqrt{\frac{M}{r^{3}}},
$$

where the second equality comes from $\dot{J}_{s}=0$ and eq. (34), and the third equality follows from (11). It is of interest to note that in general relativity, quasi-equilibrium does not require the angular frequency of the figure, $\Omega=\dot{\phi}$, to be equal to the orbital angular frequency $\dot{\theta}$; rather, $\Omega=\left(M / r^{3}\right)^{1 / 2}$, independent of the spin of the $\mathrm{BH}$.

The pressure terms in equations (31)-(33) can be conveniently rewritten in terms of the dynamic variables (LRS2):

$$
\frac{5 k_{1}}{n \kappa_{n}} \frac{P_{c}}{\rho_{c}}=\frac{m}{q_{n} R_{0}}\left(\frac{R_{0}}{R}\right)^{3 / n} \text { with } R=\left(a_{1} a_{2} a_{3}\right)^{1 / 3} .
$$


For $n \rightarrow 0$, we have $k_{1} / n \rightarrow 2 / 5$ and $\kappa_{n} \rightarrow 1$, so that

$$
\frac{5 k_{1}}{n \kappa_{n}} \frac{P_{c}}{\rho_{c}} \rightarrow \frac{2 P_{c}}{\rho_{c}} .
$$

For an incompressible ellipsoid, volume is conserved, $R^{3}=a_{1} a_{2} a_{3}=R_{0}^{3}$; Differentiating this with respect to $\tau$ gives $\sum_{i} \dot{a}_{i} / a_{i}=0$. A second derivative yields $\sum_{i} \ddot{a}_{i} / a_{i}=\sum_{i}\left(\dot{a}_{i} / a_{i}\right)^{2}$, which maybe combined with eqs. (31)-(33) to give

$$
\frac{2 P_{c}}{\rho_{c}}=\left(\sum_{i} \frac{1}{a_{i}^{2}}\right)^{-1}\left[-2\left(\Omega^{2}+\Lambda^{2}\right)+2 \Omega \Lambda\left(\frac{a_{2}}{a_{1}}+\frac{a_{1}}{a_{2}}\right)+4 \pi \bar{\rho}+\sum_{i}\left(\frac{\dot{a}_{i}}{a_{i}}\right)^{2}\right],
$$

where we have used the relations $A_{1}+A_{2}+A_{3}=2$ and $k_{11}+k_{22}+k_{33}=0$. Of course, for quasi-equilibrium, we have $\dot{a}_{i}=0$.

\section{Dimensionless Equations and Scaling Relations}

Before presenting our results on specific binary models, it is useful to cast the equations

into a dimensionless form. This will shed light on how our results depend on various parameters ( $\left.m, M, a, R_{0}\right)$ of the system. We define the following set of dimensionless variables:

$$
\begin{aligned}
\hat{a}_{i} & =\frac{a_{i}}{R_{0}} \\
\hat{\Omega} & =\Omega\left(\frac{m}{R_{0}^{3}}\right)^{-1 / 2}, \\
\hat{\Lambda} & =\Lambda\left(\frac{m}{R_{0}^{3}}\right)^{-1 / 2}, \\
\hat{c}_{i j} & =c_{i j}\left(\frac{m}{R_{0}^{3}}\right)^{-1} .
\end{aligned}
$$

Since the tidal radius is expected to be of order $R_{0}(M / m)^{1 / 3}$, we define the dimensionless orbital radius as

$$
\hat{r} \equiv \frac{r}{R_{0}}\left(\frac{m}{M}\right)^{1 / 3}
$$

In terms of these dimensionless variables, equations (31)-(33) can be written in the following form (for quasi-equilibrium):

$$
\begin{aligned}
0 & =\hat{a}_{1}\left(\hat{\Omega}^{2}+\hat{\Lambda}^{2}\right)-2 \hat{a}_{2} \hat{\Omega} \hat{\Lambda}-\frac{3}{2} A_{1} \hat{a}_{1}\left(\frac{R_{0}}{R}\right)^{3}+\frac{1}{\hat{a}_{1} q_{n}}\left(\frac{R_{0}}{R}\right)^{3 / n}-\hat{c}_{11} \hat{a}_{1} \\
0 & =\hat{a}_{2}\left(\hat{\Omega}^{2}+\hat{\Lambda}^{2}\right)-2 \hat{a}_{1} \hat{\Omega} \hat{\Lambda}-\frac{3}{2} A_{2} \hat{a}_{2}\left(\frac{R_{0}}{R}\right)^{3}+\frac{1}{\hat{a}_{2} q_{n}}\left(\frac{R_{0}}{R}\right)^{3 / n}-\hat{c}_{22} \hat{a}_{2}, \\
0 & =-\frac{3}{2} A_{3} \hat{a}_{3}\left(\frac{R_{0}}{R}\right)^{3}+\frac{1}{\hat{a}_{3} q_{n}}\left(\frac{R_{0}}{R}\right)^{3 / n}-\hat{c}_{33} \hat{a}_{3}
\end{aligned}
$$


where $R / R_{0}=\left(\hat{a}_{1} \hat{a}_{2} \hat{a}_{3}\right)^{1 / 3}$, and

$$
\hat{\Omega}=\hat{r}^{-3 / 2} .
$$

By making (40) and (41) dimensionless, the equations for the $n=0$ case may also be written in a dimensionless form. The dimensionless circulation is

$$
\hat{\mathcal{C}}=\frac{\mathcal{C}}{\left(m^{3} R_{0}\right)^{1 / 2}}=\frac{\kappa_{n}}{5}\left[\left(\hat{a}_{1}^{2}+\hat{a}_{2}^{2}\right) \hat{\Lambda}-2 \hat{a}_{1} \hat{a}_{2} \hat{\Omega}\right] .
$$

In Newtonian theory, $\hat{c}_{i j}$ depends only on $\hat{r}$, thus for given $\hat{r}$ and $\hat{\mathcal{C}}$, eqs. (47)-(51) completely determine the solution. However, this is not the case in general relativity since $\hat{c}_{i j}$ depends not just on $\hat{r}$. For example,

$$
\hat{c}_{11}=\frac{1}{\hat{r}^{3}}\left(-2-3 \frac{K}{r^{2}}\right),
$$

(The Newtonian case corresponds to $K=0$ ). It is easy to see that relativistic effect enters through the combination $K / r^{2}$, which can be expressed as

$$
\frac{K}{r^{2}}=\frac{\left[(r / M)^{1 / 2}-\hat{a}\right]^{2}}{(r / M)^{2}-3(r / M)+2 \hat{a}(r / M)^{1 / 2}},
$$

where $\hat{a} \equiv a / M$. Therefore, in addition to the dimensionless Kerr parameter $\hat{a}$, one could introduce another "parameter", $r / M$, which specifies how relativistic the orbit is. But of course this is not really a parameter, since $r$ varies as the binary evolves. A more useful parameter is

$$
\hat{R}_{0} \equiv \frac{R_{0}(M / m)^{1 / 3}}{M}=\frac{R_{0}}{m}\left(\frac{m}{M}\right)^{2 / 3}
$$

Roughly speaking, $\hat{R}_{0}$ is the ratio of the tidal radius and the horizon radius of the $\mathrm{BH}$. This parameter is convenient because it depends only on the mass and radius of the star and the mass of the $\mathrm{BH}$. When $\hat{R}_{0} \gg 1$, we expect that the binary will exist only in the Newtonian regime (i.e., the star will be disrupted before it enters into a relativistic orbit). But when $\hat{R}_{0}$ is small (of order unity), then the tidal disruption will be affected by general relativity. Note that $r / M$ can be expressed in terms of $\hat{r}$ and $\hat{R}_{0}$ as

$$
\frac{r}{M}=\hat{R}_{0} \hat{r}
$$

Thus for given $n, \hat{r}, \hat{\mathcal{C}}, \hat{a}$ and $\hat{R}_{0}$, an equilibrium binary model is uniquely determined.

\section{Equilibrium Binary Models and Tidal Limits}

As discussed in $\S 2$, in the absence of viscosity, fluid circulation is conserved; gravitational radiation reaction, being a potential force, also preserves the circulation. In a coalescing binary, 
we can determine the the relative importance of viscosity by comparing the orbital decay time $t_{d}=|r / \dot{r}|$ and the tidal synchronization time $t_{\text {syn }}$ (Bildsten \& Cutler 1992). The ratio is

$$
\frac{t_{\mathrm{syn}}}{t_{d}} \simeq\left(\frac{10}{\sin 2 \alpha}\right)\left(\frac{m}{R_{0}}\right)^{5 / 2}\left(\frac{M}{m}\right)^{2 / 3} \hat{r}^{1 / 2},
$$

where $\alpha$ is the tidal lag angle, and is proportional to the viscosity. Kochanek (1992) and Bildsten \& Cutler (1992) showed that $t_{\mathrm{syn}} / t_{d}$ is greater than unity for coalescing NS-BH binary. Using microscopic viscosity for white dwarf matter (Nandkumar \& Pethick 1984 and references therein) we can also show that the inequality $t_{\mathrm{syn}} / t_{d} \gg 1$ is well satisfied for WD-BH binaries. Thus for the types of systems (NS-BH and WD-BH) of interest in this paper, the binary will likely evolve along a quasi-equilibrium sequence with constant $\mathcal{C}$. We shall therefore focus on such models in this section.

\subsection{Roche-Riemann Model: Irrotational Equilibrium Sequences}

For the irrotational sequence, $\mathcal{C}=0$, the star has zero spin at large orbital radii $\left(\Omega_{s}=0\right)$, and equation (51) implies $\Lambda=2 a_{1} a_{2} \Omega /\left(a_{1}^{2}+a_{2}^{2}\right)$. In Figure 2 , four equilibrium sequences are depicted illustrating the effect of general relativity. Note that it is most convenient to parameterize the sequence in terms of $a_{2} / a_{1}$ since $\hat{r}$ is double-valued. We see that the star becomes more distorted as $\hat{r}$ decreases. At the same time, its central density $\rho_{c}$ decreases. This implies that the tidal field stablizes the star against collapse. Physically, this stablization arises from that fact that the self-gravity of a distorted object is weaker, and the star expands in order to satisfy hydrostatic equilibrium (see, e.g., Lai 1996; LRS1; Brady \& Hughes 1997; Wiseman 1997; Baumgarte et al. 1998; Thorne 1998; Flanagan 1998; Bonazzola et al. 1999a,b).

For each sequence, there exists a turning point, corresponding to the tidal limit, $r_{\text {tide }}$, below which no equilibrium solution is possible. From Fig. 2 , we see that stars with finite $\hat{R}_{0}$ 's are tidally disrupted at a larger $\hat{r}$ than purely Newtonian stars (with $\hat{R}_{0} \rightarrow \infty$ ), an incarnation of the old adage that GR means "stronger" gravity. We also see that the tidal limit $\hat{r}_{\text {tide }}$ is smaller for positive finite $\hat{a}$ (i.e., the orbit is prograde with respect to the $\mathrm{BH}$ spin), while $\hat{r}_{\text {tide }}$ is larger for more negative $\hat{a}$. This effect of the Kerr parameter $\hat{a}$ might also be guessed from the effect it has on the innermost stable circular orbit (ISCO): The ISCO is largest for $\hat{a}=-1, r_{\text {isco }}=9 M$, and smallest for $\hat{a}=1$, $r_{\text {isco }}=M$. The same qualitative effect applies in tidal disruption; the strongest tidal gravity is seen for the $\hat{a}=-1$ case.

The dependence of the tidal limit on $\hat{a}$ can be understood by examining the tidal field (eqs. [18][21]). The spin parameter $\hat{a}$ enters the tidal field only through $K / r^{2}$. Figure 3 shows contours of constant $K / r^{2}$ in the $\hat{a}-(r / M)$ plane. Note that only the region outside the ISCO $\left(r>r_{\text {isco }}\right)$ is of physical interest. At the ISCO, it can be shown that $K / r^{2}=1 / 3$, independent of $\hat{a}$ (This coincidence was first noted by Fishbone 1973). We see from Fig. 3 that at a given $r$, the quantity $K / r^{2}$ is smaller for larger $\hat{a}$, implying that the tidal field is weaker for larger, positive $\hat{a}$. 


\subsection{Irrotational Tidal Limit}

As discussed above, the turning point in the equilibrium sequence is of special significance: it corresponds to the tidal limit (also called "Roche-Riemann limit"), $\hat{r}_{\text {tide }}$. To determine this limit, we require $\partial \hat{r} / \partial\left(a_{2} / a_{1}\right)=0$, which adds another equation to be solved together with eqs. (47)-(49).

We have calculated $\hat{r}_{\text {tide }}$ as a function of $\hat{R}_{0}$ for a series of polytropic indices, $n=\{0,0.5,1,1.5\}$ and three Kerr parameters, $\hat{a}=\{-1,0,1\}$. The results are are shown in Fig. 4 and Fig. 5. Selected numerical values are also listed in Table 1 . For given $n$ and $\hat{a}$, there is a critical $\hat{R}_{0}=\hat{R}_{0 \text {,crit }}$, for which $r_{\text {isco }}=r_{\text {tide }}$, i.e., the inner-most stable circular orbit (ISCO) coincides with the tidal radius.

For $\hat{R}_{0}<\hat{R}_{0, \text { crit }}$, the binary encounters ISCO before tidal disruption; In this case, tidal disruption can still occur outside the BH's event horizon, but it takes place on a plunging orbit rather than on a circular orbit as in the $\hat{R}_{0}>\hat{R}_{0 \text {,crit }}$ case. Our results in Table 1 and Figs. 4-5 therefore have been truncated at $\hat{R}_{0}=\hat{R}_{0, \text { crit }}$. Note that for $\hat{R}_{0}=\hat{R}_{0, \text { crit }}(\hat{a})$ (so that tidal disruption occurs at ISCO), the tidal limit $\hat{r}_{\text {tide }}$ is independent of $\hat{a}$. This is because in the framework of our calculations, general relativistic effects come into the equations only through $K / r^{2}$, which is equal to $1 / 3$ (independent of $\hat{a}$ ) when evaluated at $r_{\text {isco }}(\hat{a})$ (see Fig. 3 ).

Figure 6 depicts the central density $\rho_{c}$ and the distortion $\left(\alpha=a_{2} / a_{1}\right.$ or $\left.a_{3} / a_{1}\right)$ of the star at the tidal limit as a function of $\hat{R}_{0}$. Only the $n=1$ results are shown as an example. We see that $\rho_{\text {c,tide }}$ and $\alpha_{\text {tide }}$ (evaluated at $r=r_{\text {tide }}$ ) are rather insensitive to $\hat{R}_{0}$ and $\hat{a}$, although the values of $\hat{r}_{\text {tide }}$ for different $\hat{R}_{0}$ and $\hat{a}$ can differ by as much as $10 \%$ (see Table 1 and Figs. 4-5).

\subsection{The Effect of Initial Spin}

When the star has an intrinsic spin $\Omega_{s}$ at large orbital radii, its (conserved) circulation is finite. Since at $r \rightarrow \infty$, the star is axisymmetric $\left(a_{1}=a_{2}\right)$, and the figure rotation $\Omega \rightarrow 0$, we have (see eq. [51])

$$
\hat{\mathcal{C}}=-\frac{2}{5} \kappa_{n} \hat{a}_{1}^{2} \hat{\Omega}_{s}
$$

where we have identified $\Omega_{s}=-\Lambda(r \rightarrow \infty)$. Note that when $\Omega_{s}$ is positive (i.e., the stellar spin is in the same direction as the orbital angular momentum), $\mathcal{C}$ is negative. The maximum spin that a uniformly rotating star can sustain without shedding mass from its equator is given by (e.g., Friedman, Ipser \& Parker 1986; Cook, Shapiro \& Teukolsky 1992)

$$
\hat{\Omega}_{s}=\frac{\Omega_{s}}{\left(m / R_{0}^{3}\right)^{1 / 2}} \lesssim 0.6 .
$$

For a given $\hat{\Omega}_{s}$, the dimensionless circulation $\mathcal{C}$ can be calculated by using the equations for compressible Maclaurin spheroid (LRS1).

To illustrate the dependence of the tidal limit on the initial stellar spin, Figure 7 shows $\hat{r}_{\text {tide }}$ as a function $\hat{R}_{0}$ for $n=0.5, \hat{a}=0$ and several different values of $\hat{\Omega}_{s}$. For negative $\hat{\Omega}_{s}$ (positive $\hat{\mathcal{C}}$ ), 
the internal rotation $\Lambda$ is larger (compared to the $\hat{\mathcal{C}}=0$ case), and this induces larger distortion to the star, and thus the star gets disrupted at a larger radius. For small, positive $\hat{\Omega}_{s}$, the internal rotation $\Lambda$ is reduced, and thus $\hat{r}_{\text {tide }}$ is smaller. But as $\hat{\Omega}_{s}$ becomes larger $\left(\hat{\Omega}_{s} \gtrsim 0.3\right), \hat{\Lambda}$ also gets larger and $\hat{r}_{\text {tide }}$ is increased over the $\hat{\Omega}_{s}=0$ value.

\subsection{Roche Model: Corotating Binaries}

Although it is likely that equilibrium sequences with constant circulation should describe the quasi-static evolutionary phase of NS-BH binaries (see the beginning of $\S 4$ for discussion), some degree of spin-orbit synchronization can still be achieved at small orbital radii through an anomalously high (turbulent) viscosity (so that $\sin 2 \alpha$ is not much less than unity). This is especially true for WD-BH binaries, for which corotating models (with $\Lambda=0$ ) may be relevant. For completeness, Table 2 lists selected values of the tidal limit (of "Roche limit") as a function of $\hat{R}_{0}$ and $\hat{a}$ for polytropic index $n=0$ and $n=1.5$. For the incompressible model $(n=0)$, our result agrees with that of Fishbone (1973) (although a different parametrization was adopted in Fishbone's paper). Similar to the irrotational models, we find that the GR effect makes $\hat{r}_{\text {tide }}$ larger than the Newtonian value, and that $\hat{r}_{\text {tide }}$ is larger for $\hat{a}=-1$ than for $\hat{a}=1$.

\section{Tidal Effect on the Orbit}

The previous sections are based on the assumption that the star $m$ moves in a geodesic of the $\mathrm{BH}$ metric. In reality, there are two corrections to the geodesic. First, even if $m$ were a point mass, its finite mass can modify the spacetime of the system. For $r \gg M+m$, the problem can be solved by post-Newtonian expansion (e.g., Blanchet et al. 1998 and references therein) or BH perturbation theory (e.g., Mino et al. 1997); when $r$ is comparable to $M+m$, nonperturbative techniques are needed (see Kidder, Will \& Wiseman 1993, Buonanno \& Damour 1998 and references therein) and definitive result will only come from numerical relativity. Second, there is a correction due to the finite size of the star. Here we focus on the second correction, i.e., the tidal effect on the orbit.

The tidal interaction energy is given by

$$
W_{\text {tide }}=-\frac{M}{2 r^{3}}\left(2 I_{11}-I_{22}-I_{33}\right)-\frac{3 M}{2 r^{3}}\left(\frac{K}{r^{2}}\right)\left(I_{11}-I_{33}\right)
$$

(see eq. [29]). The dimensionless ratio

$$
\delta=\frac{W_{\text {tide }}}{(-M m / r)}=\frac{\kappa_{n}}{10}\left(\frac{m}{M}\right)^{2 / 3} \frac{1}{\hat{r}^{2}}\left[\left(2 \hat{a}_{1}^{2}-\hat{a}_{2}^{2}-\hat{a}_{3}^{2}\right)+3\left(\frac{K}{r^{2}}\right)\left(\hat{a}_{1}^{2}-\hat{a}_{3}^{2}\right)\right],
$$

measures the correction of the tidal effect on the orbital motion (deviation from geodesic) ${ }^{2}$. Since

\footnotetext{
${ }^{2}$ In Newtonian theory, the orbital angular freqency is given by $\Omega=\left[(M+m) / r^{3}\right]^{1 / 2}(1+3 \delta / 2)$.
} 
$\left(2 \hat{a}_{1}^{2}-\hat{a}_{2}^{2}-\hat{a}_{3}^{2}\right)$ and $\left(\hat{a}_{1}^{2}-\hat{a}_{3}^{2}\right)$ are of order $1 / \hat{r}^{3}$, and $K / r^{2}$ is of order $M / r<1$, we see that $\delta$ scales as $1 / \hat{r}^{5}$. Note that even near the tidal radius $(\hat{r} \sim 1), \delta$ can be quite small if $m / M$ is small.

In the absence of tidal coupling, the dynamical instability limit $r_{\mathrm{dyn}}$ of the orbit is located at ISCO. We assume that $r_{\text {isco }}$ is given by the standard $M \gg m$ result. (When $m / M$ is finite, see Buonanno \& Damour 1999 and references therein.) Tidal coupling is expected to shift $r_{\text {dyn }}$ outward compared to $r_{\mathrm{ISCO}}$ (see Lai \& Wiseman 1996). To estimate the correction, $\left(r_{\text {dyn }}-r_{\text {isco }}\right)$, we write the total equilibrium energy of the binary system as

$$
\mathcal{E}=m E+\Delta \mathcal{E},
$$

where $E$ is the energy per unit mass of a test particle orbiting a Kerr BH (see eq. [9]), and $\Delta \mathcal{E}$ is the correction due to the finite size of the star. Minimizing $\mathcal{E}$ would give $r_{\text {dyn }}$. Unfortunately there is no unambiguous way of writing down the total energy $\mathcal{E}$ in general relativity. However, we can still attempt to determine how $\left(r_{\text {dyn }}-r_{\text {isco }}\right)$ scales with various parameters of the system. From Newtonian consideration, $\Delta \mathcal{E}$ is certainly of order $\left(-W_{\text {tide }}\right)$ - The negative sign arises because the tidal attraction increases the kinetic energy of the orbital motion, which more than compensates for the neative tidal potential energy. We therefore set $\Delta \mathcal{E}=-\beta W_{\text {tide, }}$, with $\beta$ of order unity. Assuming that $r_{\text {dyn }}$ is close to $r_{\text {isco }}$, we find ${ }^{3}$ :

$$
\epsilon \equiv \frac{r_{\mathrm{dyn}}-r_{\text {isco }}}{M}=C \beta \kappa_{n} \hat{R}_{0}^{2}\left(\frac{m}{M}\right)^{2 / 3}\left(2 \hat{a}_{1}^{2}-\hat{a}_{2}^{2}-\hat{a}_{3}^{2}\right)_{\mathrm{isco}}
$$

where $\left(2 \hat{a}_{1}^{2}-\hat{a}_{2}^{2}-\hat{a}_{3}^{2}\right)$ is evaluated at $r=r_{\text {isco. }}$. The constant $C$ depends on $r_{\text {isco }} / M$ and $d^{2} E / d r^{2}$ (evaluated at $r_{\text {isco }}$ ): $C=0.116$ for $\hat{a}=0.998$, and 0.106 for $\hat{a}=0$ and 0.077 for $\hat{a}=-1$. Although we cannot determine the precise value of $\beta$, we expect the scaling in eq. (62) to be valid in general.

The biggest correction to $r_{\text {dyn }}$ occurs when tidal disruption occurs just inside the ISCO. For example, when $\hat{R}_{0}=2, a=0$ and $n=0$, we have $\epsilon=0.53 \beta(m / M)^{2 / 3}$ (assuming $m / M$ is small).

\section{Possible Applications to Gamma-Ray Bursts}

In this section we discuss possible applications of our results to Gamma-Ray Bursts (GRBs).

\subsection{Black Hole - Neutron Star Binaries}

Coalescence of BH-NS binaries has frequently been invoked as the central engine of GRBs (e.g., Narayan, Paczynski \& Piran 1992; Meszaros, Rees \& Wijers 1998; Piran 1998). It is expected

\footnotetext{
${ }^{3}$ In taking derivative of $W_{\text {tide }}$ with respect to $r$, we have assumed that $K / r^{2}$ is roughly constant, which we have checked to be reasonable as long as $\hat{a}$ is not too close to 1 . Also we have found that $\left(2 \hat{a}_{1}^{2}-\hat{a}_{2}^{2}-\hat{a}_{3}^{2}\right)$ is approximately equal to twice of $\left(a_{1}^{2}-a_{3}^{2}\right)$, and both scale approximately as $r^{-3}$. Relaxing these approximations would give rise to a factor of order unity which we can absorb into the definition of $\beta$.
} 
that a thick disk or torus will result from the disruption of the NS. To account for the temporal properties of observed GRBs, the disk must be long-lived (compared to the orbital timescale) (e.g., Meszaros, Rees \& Wijers 1998; van Putten 1999), and therefore the tidal disruption must occur outside the ISCO, i.e., $r_{\text {tide }}>r_{\text {ISCO }}$. Approximating the NS as a $n=0.5$ polytrope, we find that the tidal radius for critical $\hat{R}_{0}=\hat{R}_{0, \text { crit }}$ (where $r_{\text {tide }}=r_{\text {isco }}$ ) is $\hat{r}_{\text {tide }}=2.56$ (see Table 1 ). Therefore the condition $r_{\text {tide }}>r_{\text {isco }}$ translates to

$$
\frac{M}{m}<\left(\frac{M}{m}\right)_{\text {crit }}=3.12\left(\frac{R_{0}}{5 m}\right)^{3 / 2}\left(\frac{6 M}{r_{\text {isco }}}\right)^{3 / 2} .
$$

For canonical neutron star parameters $\left(m=1.4 M_{\odot}, R_{0}=10 \mathrm{~km}\right.$, corresponding to $\left.R_{0} / m \simeq 4.84\right)$, tidal disruption occurs outside ISCO only if $M \lesssim 4.4 M_{\odot}($ for $\hat{a}=0 \mathrm{BH})$ or $M \lesssim 64 M_{\odot}($ for $\hat{a}=1$ $\mathrm{BH})$.

We can calculate the maximum energy available in the disk following a disruption by comparing

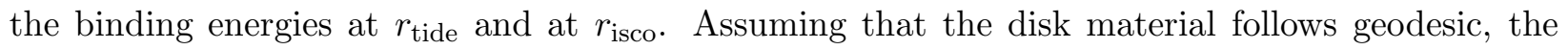
maximum energy release per unit mass of accreting gas is given by

$$
\Delta E=E\left(r_{\text {tide }}\right)-E\left(r_{\text {isco }}\right),
$$

where $E(r)$ is given by eq. (9). Note that $\Delta E$ depends only on $\hat{R}_{0}$. Figure 8 depicts $\Delta E$ as a function of the "effective" mass ratio $(M / m)\left(R_{0} / 5 m\right)^{-3 / 2}=\left(5 / \hat{R}_{0}\right)^{3 / 2}$ for different value of $\mathrm{BH}$ spin parameter $\hat{a}(\hat{a}=-1,0,0.5,0.9,0.998$ and $a \rightarrow 1$; Note that $\hat{a}=0.998$ corresponds to the maximum value of $\hat{a}$ that can be achieved in accretion; Thorne 1974). Clearly, $\Delta E=0$ when $M / m=(M / m)_{\text {crit }}$ since $r_{\text {tide }}=r_{\text {isco. }}$. When $(M / m) \ll(M / m)_{\text {crit }}, \Delta E$ approaches the standard radiation efficiency for thin disk, $\Delta E=1-E\left(r_{\text {isco }}\right)$. The dependence of $\Delta E$ on $\hat{a}$ mainly comes from $r_{\text {isco }}$ since $r_{\text {tide }}$ is relatively insensitive to $\hat{a}$ (see Figs. $4-5$ and Table 1). Note that our calculation is valid only when $M / m \gg 1$, so the results for $M / m$ less than a few should be considered suggestive only.

Recent observations indicate that some GRBs are highly energetic, with the apparent emitted energy in gamma rays reaching $10^{53}$ erg (e.g., Kulkarni et al. 1999 and references therein). Uncertainty in beaming precludes accurate determination of the true gamma ray energy. It has been suggested that the total energy in the fireball may be as high as $10^{54} \mathrm{erg}$ (e.g., Kumar 1999). If this number holds up, we may rule out NS-BH binary coalescence as the central engine of GRBs (see Fig. 8).

\subsection{Black Hole - White Dwarf Binaries}

For a BH-WD binary, tidal disruption occurs outside ISCO $\left(r_{\text {tide }}>r_{\text {isco }}\right)$ when

$$
\frac{M}{m}<\left(\frac{M}{m}\right)_{\text {crit }}=2.18 \times 10^{5}\left(\frac{R_{0}}{10^{4} m}\right)^{3 / 2}\left(\frac{6 M}{r_{\text {isco }}}\right)^{3 / 2},
$$


where we have adopted the $n=1.5$ polytropic model for the WD, and used $\hat{r}_{\text {tide }}=2.173$ (see Table 1) for $\hat{R}_{0}=\hat{R}_{0, \text { crit }}$. Although there is no evidence for massive BHs with mass around $10^{5} M_{\odot}$ (e.g., Richstone et al. 1998), the observational constraint on such BHs is also weak. If they do exist, they may capture WDs and the merger of the BH-WD binary may lead to a GRB (R. Blandford 1997, private communication). For $(M / m) \ll(M / m)_{\text {crit }}$, a Newtonian description for tidal disruption is adequate; but when $M / m$ is close to $(M / m)_{\text {crit }}$, a general relativistic treatment is necessary. Since $M \gg m$ is well satisfied, the model presented in this paper should provide an accurate description of BH-WD binaries.

Figure 8 shows the maximum energy available in a BH-WD tidal disruption. Unlike BH-NS binaries, the limiting value of $\Delta E$, corresponding to the thin disk limit, $\Delta E=1-E\left(r_{\text {isco }}\right)$, can be easily achieved for a large range of mass ratios.

\section{Discussion}

Quasi-equilibrium models of neutron star binaries provide an accurate description of the late stage of inspiralling binary stars prior to the dynamical merger. While quasi-equilibrium NS-NS binary models (the so-called "Darwin-Riemann problems") have been successfully constructed in general relativity (see Bonazzola et al. 1999a,b for a review), only Newtonian models of NS-BH binaries have been studied so far (see Uryu \& Eriguchi 1999). The semi-analytic Roche-Riemann models studied in this paper provide useful insight on the relativistic tidal effects in coalescing NS-BH and WD-BH binaries.

We note that our model is exact when the following conditions are satisfied: (i) $M \gg m$ [so that the spacetime is given by the Kerr metric of the $\mathrm{BH}(\S 2)$, and the tidal correction to the orbit is negligible (§5)]; (ii) $R_{0} / m \gg 1$ (so that we can treat the fluid star as a Newtonian object); (iii) The radius of curvature $\left(r^{3} / M\right)^{1 / 2} \gg R_{0}$ [so that high-order tidal effect can be neglected (§2); At the $r=r_{\text {tide }} \sim(M / m)^{1 / 3} R_{0}$, this condition translates to $\left(R_{0} / m\right)^{1 / 2} \gg 1$, and is equivalent to condition (ii)]; (iv) $n=0$ [The ellipsoid ansatz is exact when the external force is a linear function of spatial coordinates, as required by (iii), and when the fluid is incompressible (Chandrasekhar 1969)]. Clearly, for WD-BH binaries, conditions (i)-(iii) are well-satisfied, and the ellipsoid ansatz is a good approximation for polytrope of $n=1.5$ (appropriate for low-mass WD) (LRS1 ${ }^{4}$ ). Therefore our findings that general relativitic effect increases $r_{\text {tide }}$ and that $r_{\text {tide }}$ is largest for $\hat{a}=-1$ (regtrograde $\mathrm{BH})$ are expected to hold.

For NS-BH binaries, conditions (i)-(iii) are satisfied only approximately, we must apply our results with caution. In particular, post-Newtonian correction to the self-gravity is likely to be important. The height $H$ of tidal bulge on the star can be estimated from $\left(M R_{0} / r^{3}\right)\left[1+\mathcal{O}\left(K / r^{2}\right)\right] \sim$

\footnotetext{
${ }^{4}$ Note that for very compressible fluid (large $n$ ), the tidal limit corresponds to the point where mass transfer starts; see Uryu \& Eriguchi (1999).
} 
$\left(m H / R_{0}^{3}\right)\left[1+\mathcal{O}\left(m / R_{0}\right)\right]$. Setting $H \sim R$ gives the tidal radius

$$
r_{\text {tide }} \sim R_{0}\left(\frac{M}{m}\right)^{1 / 3}\left[1+\mathcal{O}\left(\frac{K}{r^{2}}\right)-\mathcal{O}\left(\frac{m}{R_{0}}\right)\right] .
$$

While relativistic tidal field (the $K / r^{2}$ term) increases $r_{\text {tide }}$, the post-Newtonian self-gravity effect

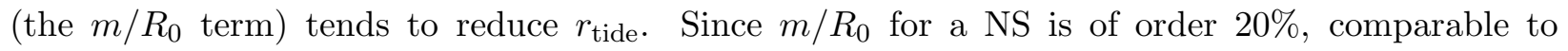

$K / r^{2}$ (which is always less than $1 / 3$; see Fig. 3 ), the post-Newtonian self-gravity effect cannot be neglected. Note that our finding that $r_{\text {tide }}$ is largest for $\hat{a}=-1$ is still expected to be valid.

P.W. acknowledges support from the NSF REU program at Cornell, and D.L. thanks the Alfred P. Sloan Foundation for a research fellowship.

\section{REFERENCES}

Baumgarte, T. W., et al. 1998, Phys. Rev. D57, 7299.

Bildsten, L., \& Cutler, C. 1992, ApJ, 400, 175.

Blanchet, L., Faye, G., Ponsot, B. 1998, Phys. Rev. D58, 124002.

Bonazzola, S, Gourgoulhon, E, \& Marck, J. A. 1999a, Phys. Rev. Lett., 82, 892.

Bonazzola, S, Gourgoulhon, E, Grandclement, P., \& Marck, J. A. 1999b, gr-qc/9909020.

Brady, P. R., \& Hughes, S. 1997, Phys. Rev. Lett., 79, 1186.

Buonanno, A., \& Damour, T. 1999, Phys. Rev. D59, 084006 (gr-qc/9811091).

Chandrasekhar, S. 1969, Ellipsoidal Figures of Equilibrium (New Haven: Yale University Press).

Cook, G. B., Shapiro, S. L., \& Teukolsky, S. A. 1992, ApJ, 398, 203.

Fishbone, L. G. 1973, ApJ, 185, 43.

Flanagan, E. E. 1998, Phys. Rev. D 5812, 4030.

Friedman, J. L., Ipser, J. R., \& Parker, L. 1986, ApJ, 304, 115.

Fryer, C. L., Woosley, S. E., Herant, M., \& Davies, M. B. 1998, astro-ph/9808094.

Janka, H.-Th., Eberl, T., Ruffert, M., \& Fryer, C. L. 1999, astro-ph/9908290.

Kidder, L. E., Will, C. M., \& Wiseman, A. G. 1993, Phys. Rev. D47, 3281.

Kluzniak, W., \& Lee, W. H. 1998, ApJ, 494, L53. 
Kumar, P. 1999, ApJ, in press (astro-ph/9907096).

Kochanek, C.S. 1992, ApJ, 398, 234.

Kulkarni, S. R., et al. 1999, Nature, 393, 389.

Lai, D. 1996, Phys. Rev. Lett., 76, 4878.

Lai, D., Rasio, F. A., \& Shapiro, S. L. 1993, ApJ Supp., 88, 205 (LRS1).

Lai, D., Rasio, F. A., \& Shapiro, S. L. 1994, ApJ, 437, 742 (LRS2).

Lai, D., \& Wiseman, A. G. 1996, Phys. Rev. D54, 3958.

Lattimer, J. M., \& Schramm, D. M. 1976, ApJ, 210, 549.

Marck, J.-A. 1983, Proc. R. Soc. Lond., A385, 431.

Mashhoon, B. 1975, ApJ, 197, 705.

Meszaros, P., Rees, M. J., \& Wijers, R.A.M.J. 1998, submitted to New Astronomy (astro$\mathrm{ph} / 9808106)$.

Mino, Y., Sasaki, M., Shibata, M., Tagoshi, H., \& Tanaka, T. 1997, Prog. Theor. Phys. Suppl., 128, 1 (gr-qc/9712057).

Misner, C., Thorne, K. S., \& Wheeler, J. A. 1973, Gravitation (San Francisco: Freeman)

Narayan, R., Paczynski, \& Piran, T. 1992, ApJ, 395, L83.

Nandkumar, R., \& Pethick, C. J. 1984, MNRAS, 209, 511.

Piran, T. 1998, Phys. Report, in press (astro-ph/9810256).

Richstone, D., et al. 1998, Nature, 395A, 14.

Shibata, M. 1996, Prog. Theo. Phys., 96, 917.

Shibata, M., \& Taniguchi, K. 1997, Phys. Rev. D56, 811.

Taniguchi, K. 1999, Prog. Theor. Phys., 101, 283 (astro-ph/9901048)

Taniguchi, K., \& Shibata, M. 1997, Phys. Rev. D56, 798.

Thorne, K. S. 1974, ApJ, 191, 507.

Thorne, K.S. 1997, in "Black Holes and Relativistic Stars" ed. R. Wald (Univ. of Chicago Press).

Thorne, K. S. 1998, Phys. Rev. D 5812, 4031. 
Uryu, K., \& Eriguchi, Y. 1999, MNRAS, 303, 329.

Van Putten, M. H. P. M. 1999, Science, 284, 115.

Wiseman, A. G. 1997, Phys. Rev. Lett., 79, 1189. 
Table 1. Tidal Limits for Irrotational Binaries

\begin{tabular}{|c|c|c|c|}
\hline \multirow[b]{2}{*}{$\hat{R}_{0}$} & \multicolumn{3}{|c|}{$\hat{r}_{\text {tide }}$} \\
\hline & $\hat{a}=-1$ & $\hat{a}=0$ & $\hat{a}=+1$ \\
\hline \multicolumn{4}{|c|}{$n=0$} \\
\hline$\infty$ & 2.502 & 2.502 & 2.502 \\
\hline 6 & 2.607 & 2.569 & 2.539 \\
\hline 4 & 2.689 & 2.611 & 2.552 \\
\hline 3.265 & 2.757 & 2.644 & 2.561 \\
\hline 3 & $\cdots$ & 2.661 & 2.565 \\
\hline 2.176 & $\ldots$ & 2.757 & 2.581 \\
\hline 2 & $\cdots$ & $\ldots$ & 2.586 \\
\hline 1 & $\ldots$ & $\ldots$ & 2.638 \\
\hline 0.363 & $\ldots$ & $\ldots$ & 2.757 \\
\hline \multicolumn{4}{|c|}{$n=0.5$} \\
\hline$\infty$ & 2.320 & 2.320 & 2.320 \\
\hline 6 & 2.430 & 2.389 & 2.357 \\
\hline 4 & 2.517 & 2.433 & 2.370 \\
\hline 3.515 & 2.560 & 2.454 & 2.375 \\
\hline 3 & $\ldots$ & 2.487 & 2.382 \\
\hline 2.344 & . & 2.560 & 2.395 \\
\hline 2 & $\cdots$ & $\ldots$ & 2.404 \\
\hline 1 & $\ldots$ & $\ldots$ & 2.455 \\
\hline 0.390 & $\ldots$ & $\ldots$ & 2.560 \\
\hline \multicolumn{4}{|c|}{$n=1$} \\
\hline$\infty$ & 2.140 & 2.140 & 2.140 \\
\hline 6 & 2.255 & 2.210 & 2.176 \\
\hline 4 & 2.349 & 2.257 & 2.190 \\
\hline 3.805 & 2.366 & 2.266 & 2.191 \\
\hline 3 & $\ldots$ & 2.315 & 2.202 \\
\hline 2.536 & $\ldots$ & 2.366 & 2.210 \\
\hline 2 & $\ldots$ & $\ldots$ & 2.223 \\
\hline 1 & $\ldots$ & $\ldots$ & 2.273 \\
\hline 0.423 & $\ldots$ & $\ldots$ & 2.366 \\
\hline
\end{tabular}


Table 1-Continued

\begin{tabular}{rccc}
\hline \hline & \multicolumn{3}{c}{$\hat{r}_{\text {tide }}$} \\
\cline { 2 - 4 }$\hat{R}_{0}$ & $\hat{a}=-1$ & $\hat{a}=0$ & $\hat{a}=+1$ \\
\hline \multicolumn{4}{c}{$n=1.5$} \\
\hline \multicolumn{4}{c}{1.960} \\
6 & 1.960 & 1.960 & 1.960 \\
4.141 & 2.083 & 2.034 & 1.997 \\
4 & $\ldots$ & 2.079 & 2.009 \\
3 & $\ldots$ & 2.085 & 2.014 \\
2.761 & $\ldots$ & 2.173 & 2.023 \\
2 & $\ldots$ & $\ldots$ & 2.026 \\
1 & $\ldots$ & $\ldots$ & 2.044 \\
0.460 & $\ldots$ & $\ldots$ & 2.173 \\
\hline
\end{tabular}

Note. $\quad-\quad \hat{R}_{0} \equiv \frac{R_{0}}{m}\left(\frac{m}{M}\right)^{2 / 3}$ ( $m, R_{0}$ are the mass and radius of the star, $M$ is the BH mass), $\hat{r}_{\text {tide }} \equiv$ $\frac{r_{\text {tide }}}{R_{0}}\left(\frac{m}{M}\right)^{1 / 3}, \hat{a}$ is the Kerr parameter of the $\mathrm{BH}$, and $n$ is the polytropic index. The last item in each column corresponds to $\hat{R}_{0}=\hat{R}_{0 \text {,crit }}$ at which $r_{\text {tide }}=r_{\text {isco }}$ or $\hat{R}_{0} \hat{r}_{\text {tide }}=$ $\left(\frac{r}{M}\right)_{\text {isco }}$. 
Table 2. Tidal Limits for Corotating Binaries

\begin{tabular}{|c|c|c|c|}
\hline \multirow[b]{2}{*}{$\hat{R}_{0}$} & \multicolumn{3}{|c|}{$\hat{r}_{\text {tide }}$} \\
\hline & $\hat{a}=-1$ & $\hat{a}=0$ & $\hat{a}=+1$ \\
\hline \multicolumn{4}{|c|}{$n=0$} \\
\hline$\infty$ & 2.455 & 2.455 & 2.455 \\
\hline 6 & 2.566 & 2.525 & 2.493 \\
\hline 4 & 2.653 & 2.570 & 2.508 \\
\hline 3.311 & 2.718 & 2.602 & 2.516 \\
\hline 3 & $\cdots$ & 2.623 & 2.521 \\
\hline 2.208 & $\ldots$ & 2.718 & 2.537 \\
\hline 2 & $\cdots$ & $\cdots$ & 2.543 \\
\hline 1 & $\cdots$ & $\ldots$ & 2.597 \\
\hline 0.368 & $\cdots$ & $\ldots$ & 2.718 \\
\hline \multicolumn{4}{|c|}{$n=1.5$} \\
\hline$\infty$ & 2.035 & 2.035 & 2.035 \\
\hline 6 & 2.148 & 2.103 & 2.069 \\
\hline 4.018 & 2.240 & 2.149 & 2.082 \\
\hline 4 & $\cdots$ & 2.150 & 2.082 \\
\hline 3 & $\ldots$ & 2.207 & 2.093 \\
\hline 2.679 & $\cdots$ & 2.240 & 2.099 \\
\hline 2 & $\cdots$ & $\cdots$ & 2.113 \\
\hline 1 & $\ldots$ & $\cdots$ & 2.160 \\
\hline 0.447 & $\ldots$ & $\ldots$ & 2.240 \\
\hline
\end{tabular}

Note. $\quad-\quad \hat{R}_{0} \equiv \frac{R_{0}}{m}\left(\frac{m}{M}\right)^{2 / 3}$, $\hat{r}_{\text {tide }} \equiv \frac{r_{\text {tide }}}{R_{0}}\left(\frac{m}{M}\right)^{1 / 3}$. The last item in each column corresponds to $\hat{R}_{0}=\hat{R}_{0, \text { crit }}$ at which $r_{\text {tide }}=r_{\text {isco }}$ or $\hat{R}_{0} \hat{r}_{\text {tide }}=\left(\frac{r}{M}\right)_{\text {isco }}$. 


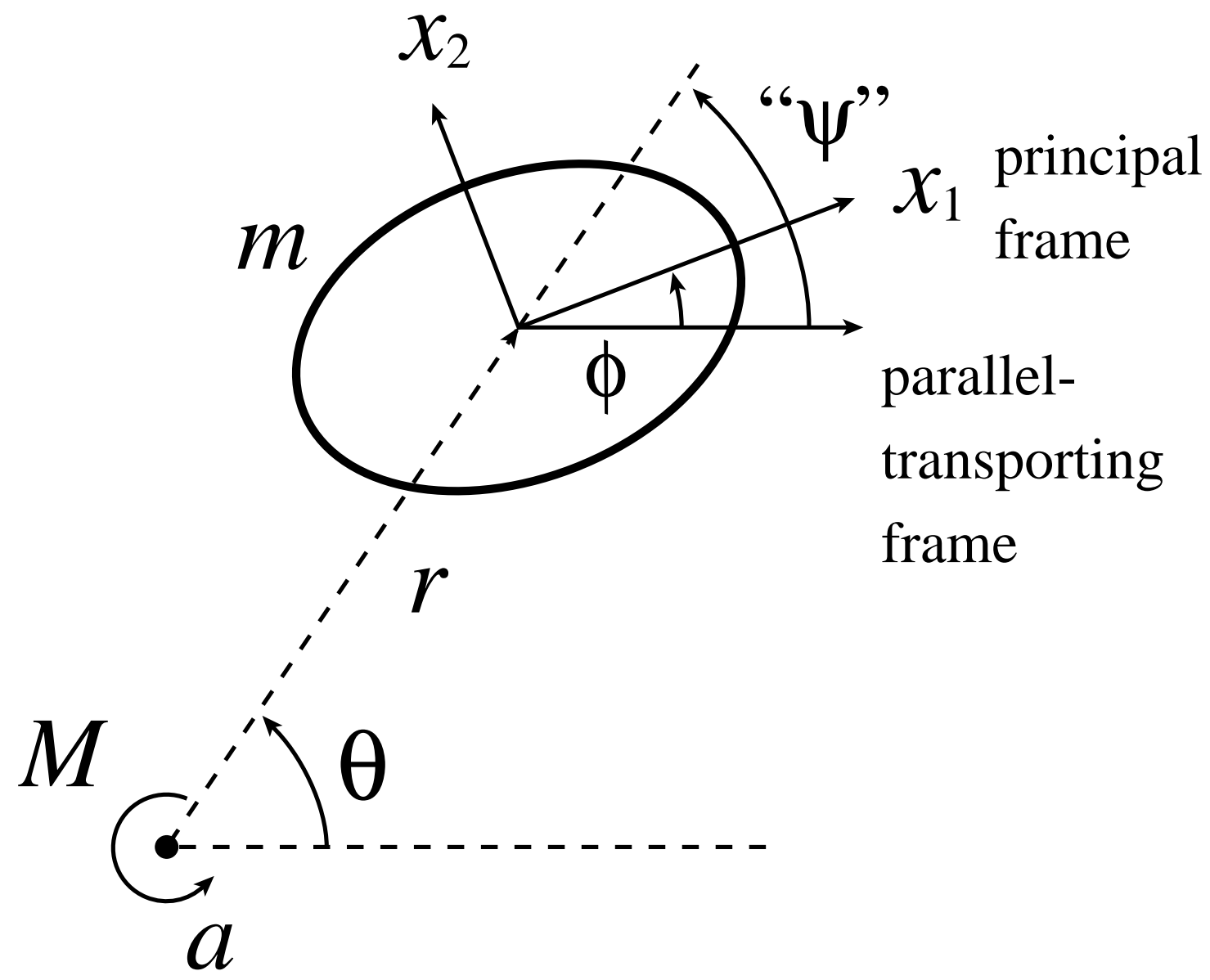

Fig. 1.- Sketch of the Roche-Riemann binary model. The star is represented by an ellipsoid with mass $m$. The $\mathrm{BH}$ has mass $M$ and Kerr parameter $a$ (The $\mathrm{BH}$ spin is aligned with the orbital angular momentum). The angle $\Psi$ is shown with quotes since the drawing is only correct in the Newtonian limit (see text). 


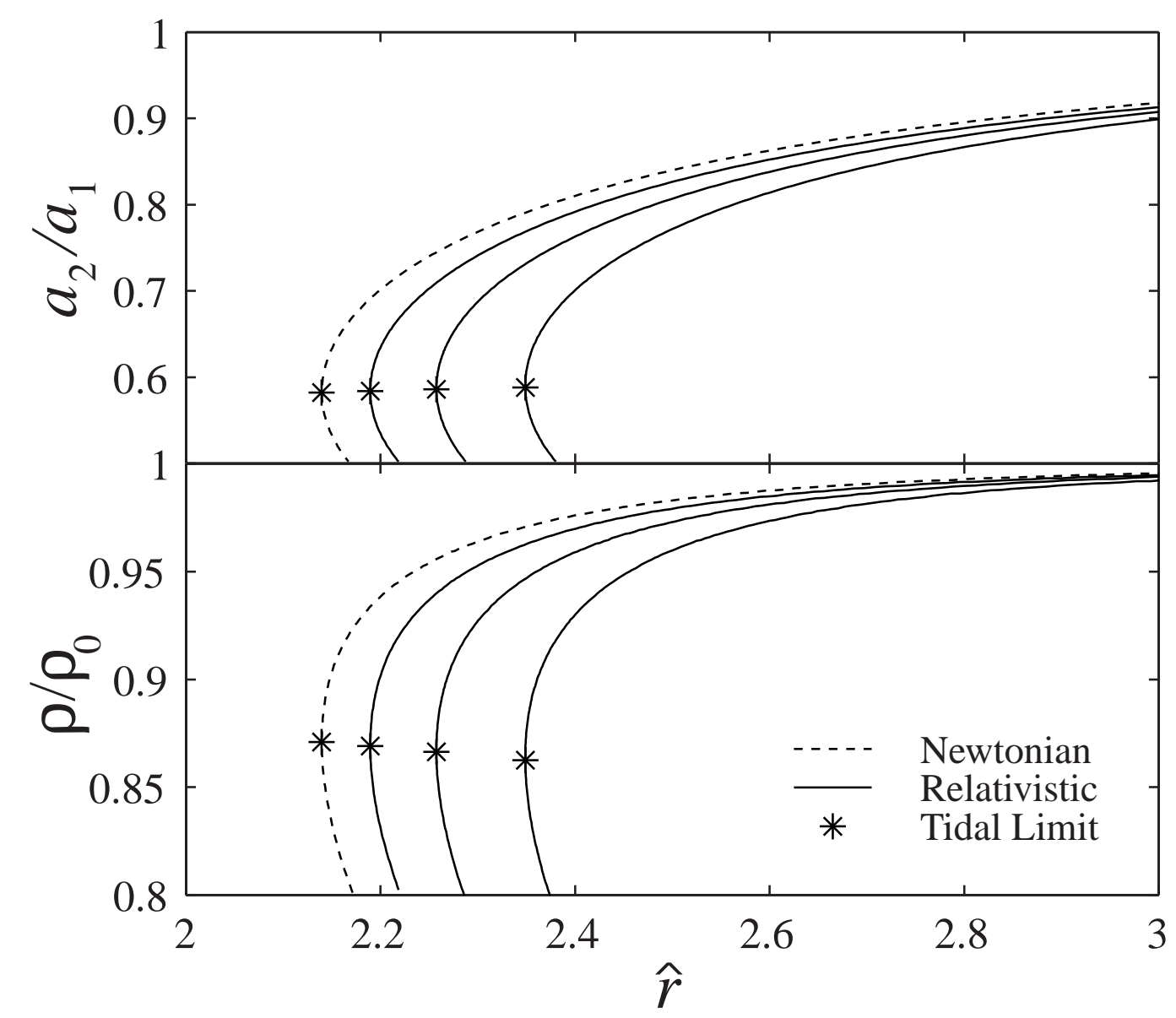

Fig. 2.- Equilibrium sequences for irrotational binaries $(\mathcal{C}=0)$. The axis ratio $a_{2} / a_{1}$ and the central density $\rho_{c}$ [in units of its value at infinite binary separation, $\rho_{0}=\rho_{c}(r \rightarrow \infty)$ ] are plotted against the dimensionless orbital separation $\hat{r}=\left(r / R_{0}\right)(m / M)^{1 / 3}$. The polytropic index is $n=1$. The dashed curves represent the Newtonian result, with $\hat{R}_{0} \rightarrow \infty$. The three solid curves include the effects of general relativity (with $\hat{a}=1,0,-1$ from left to right), all for $\hat{R}_{0}=4$. The turning point of each curve, with $\hat{r}$ reaching a minimum, is marked by an asterisk, and corresponds to the tidal limit. Note that the values of $a_{2} / a_{1}$ and $\rho_{c} / \rho_{0}$ at $\hat{r}=\hat{r}_{\text {tide }}$ are similar for different sequences, but they are not identical (see Fig. 6). 


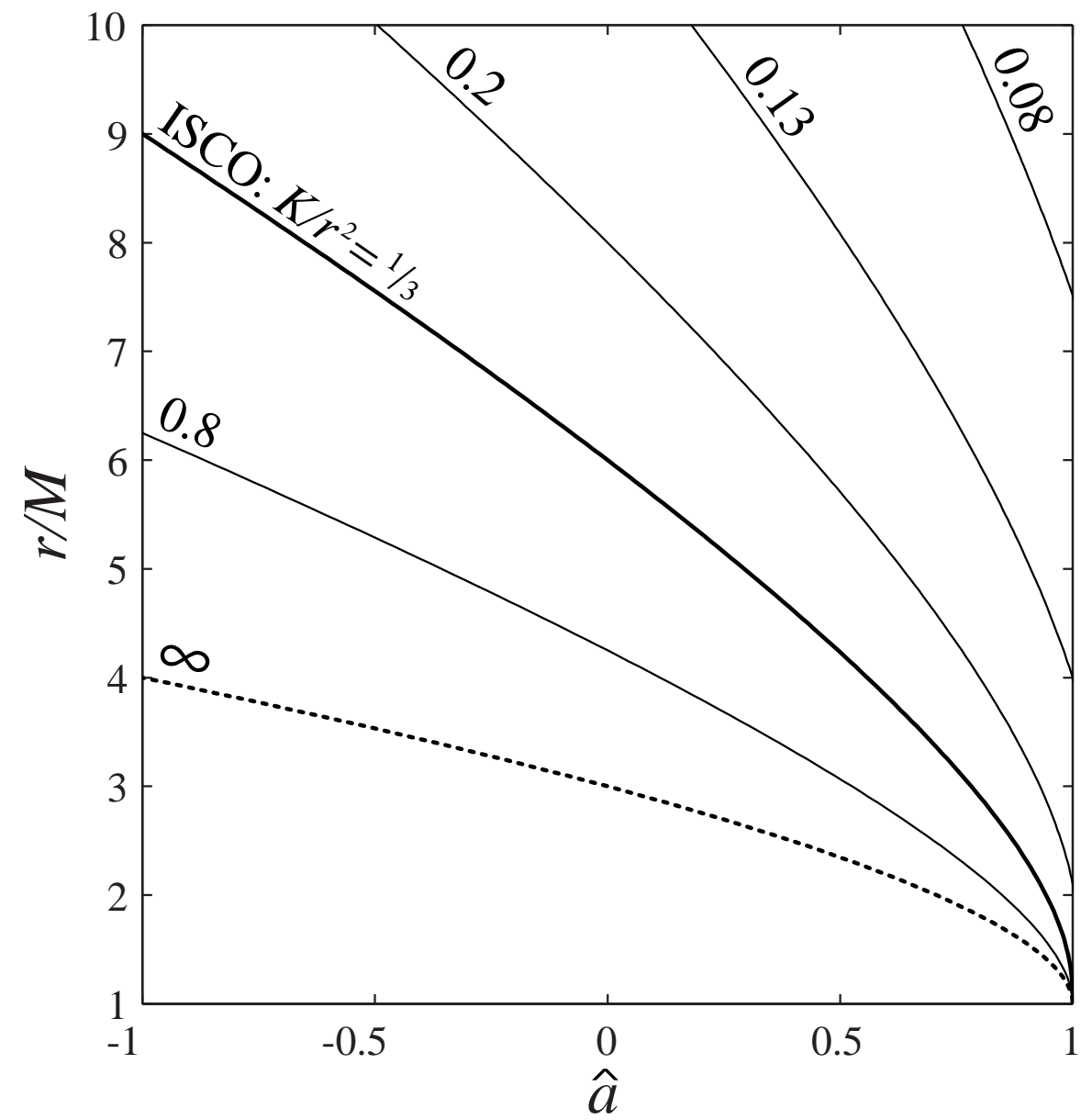

Fig. 3.- Contours of constant $K / r^{2}$ in the $\hat{a}-(r / M)$ plane. This quantity determines the magnitude of relativistic effect on the tidal distortion. The number on each curve gives the value of $K / r^{2}$. For physically reasonable $r / M$, the quantity $K / r^{2}$ is positive and decreases with increasing $\hat{a}$ and $r / M$. Note that at the innermost stable circular orbit (ISCO), $K / r^{2}=1 / 3$ is independent of $a$. Thus the heavy solid line also gives the value of $r_{\text {isco }}$ as a function of $\hat{a}$. The pole of $K / r^{2}$ (where it becomes infinite) is hidden inside the ISCO except when $\hat{a}=1$. 


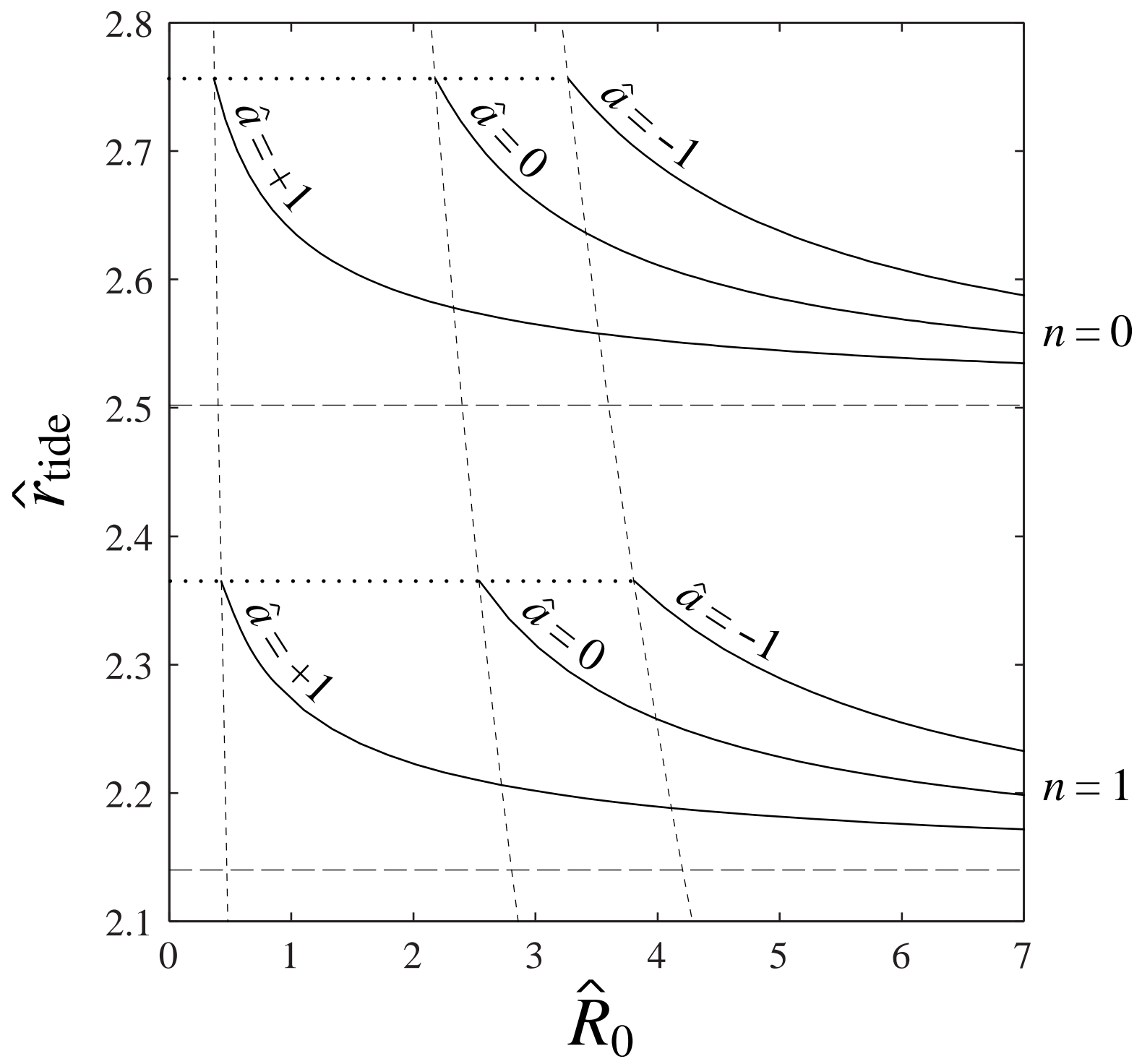

Fig. 4. - Tidal limit $\hat{r}_{\text {tide }}=\left(r_{\text {tide }} / R_{0}\right)(m / M)^{1 / 3}$ as a function of $\hat{R}_{0}=\left(R_{0} / m\right)(m / M)^{2 / 3}$ for irrotational binaries $(\mathcal{C}=0)$. The two sets of solid curves correspond to polytropic index $n=0$ and $n=1$ respectively. Results for three values of Kerr parameter $(\hat{a}=-1,0,1)$ are shown. Each solid curve is truncated at $\hat{R}_{0}=\hat{R}_{0 \text {,crit }}$, where $r_{\text {tide }}=r_{\text {isco }}$ [The short-dashed lines represent the ISCO for different Kerr parameters, i.e., $\hat{R}_{0} \hat{r}=(r / M)_{\text {isco }}$. The two horizontal dotted lines show the limiting $\hat{r}_{\text {tide }}$ at the truncation. Two additional long-dashed lines show the Newtonian limit of the tidal limit. For $n=0$, the Newtonian limit is $\hat{r}_{\text {tide }}=2.502$ and the ISCO limit is $\hat{r}_{\text {tide }}=2.757$. For $n=1$, the Newtonian limit is $\hat{r}_{\text {tide }}=2.140$ and the ISCO limit is $\hat{r}_{\text {tide }}=2.366$. 


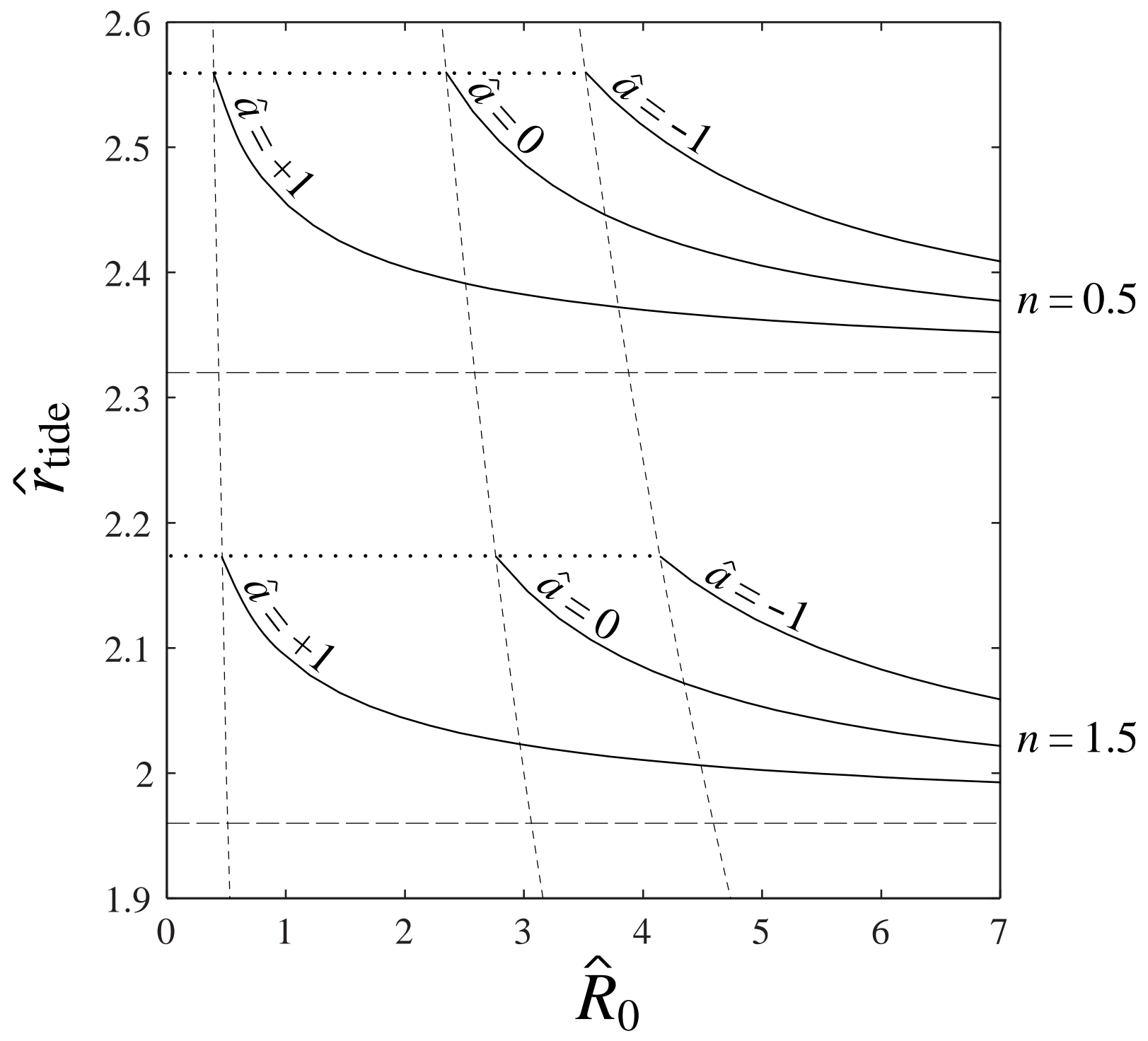

Fig. 5.- Same as Fig. 4 except for $n=0.5$ and $n=1.5$. For $n=0.5$, the Newtonian limit is $\hat{r}_{\text {tide }}=2.320$ and the ISCO limit is $\hat{r}_{\text {tide }}=2.560$. For $n=1.5$, the Newtonian limit is $\hat{r}_{\text {tide }}=1.960$ and the ISCO limit is $\hat{r}_{\text {tide }}=2.173$. 


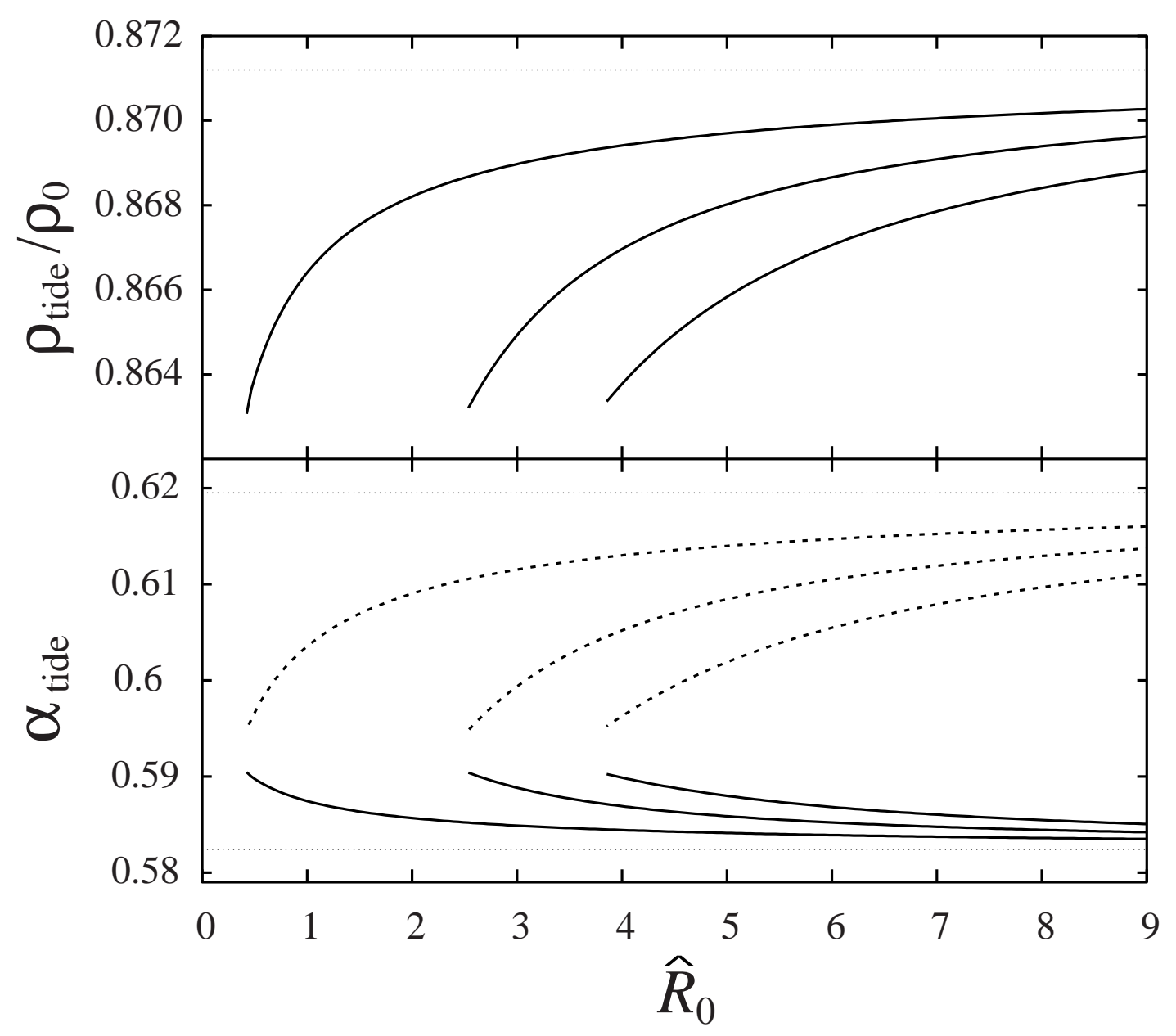

Fig. 6. - The central density [in units of $\rho_{0}=\rho_{c}(r \rightarrow \infty)$ ] and the axis ratio $\left(\alpha=a_{2} / a_{1}\right.$ or $\left.a_{3} / a_{1}\right)$ of the star at the tidal limit $\left(\hat{r}=\hat{r}_{\text {tide }}\right)$ as a function of $\hat{R}_{0}$ for $\hat{a}=\{1,0,-1\}, n=1$ and $\mathcal{C}=0$. The solid curves in the lower plot are for $\alpha \equiv a_{2} / a_{1}$ and the dashed curves are for $\alpha \equiv a_{3} / a_{1}$. From left to right, the curves are $\hat{a}=\{1,0,-1\}$. The horizontal dotted lines correspond to the Newtonian limit. 


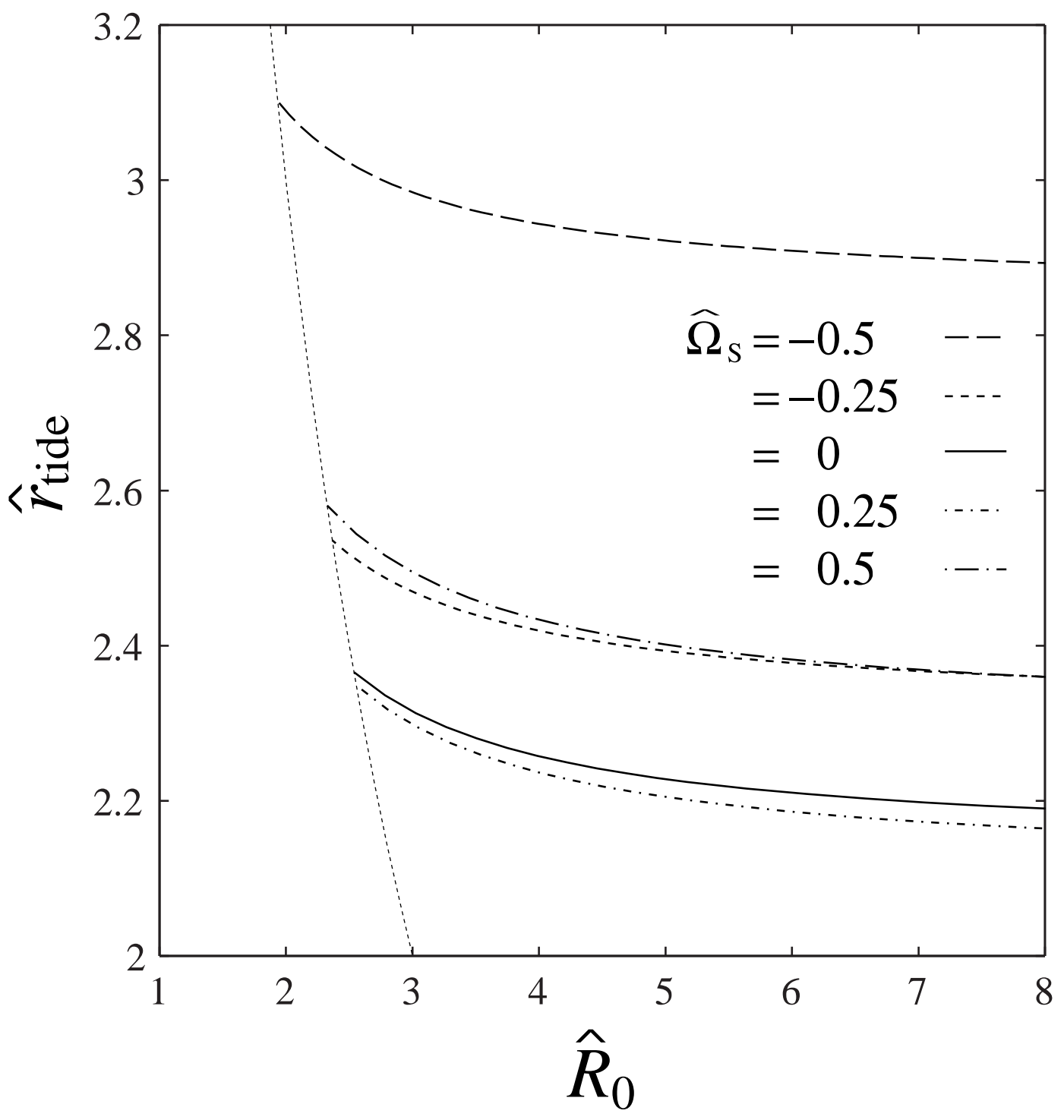

Fig. 7. - Tidal limits in models with initial rotation $\left(\hat{\Omega}_{s} \neq 0\right)$ for $n=0.5$ and $\hat{a}=0$. As in Figs. 4-5, each curve is truncated at $\hat{R}_{0}=\hat{R}_{0, \text { crit }}$, where $r_{\text {tide }}=r_{\text {isco }}$ [The short-dashed line represents the ISCO, i.e., $\hat{r} \hat{R}_{0}=(r / M)_{\text {isco }}$. 


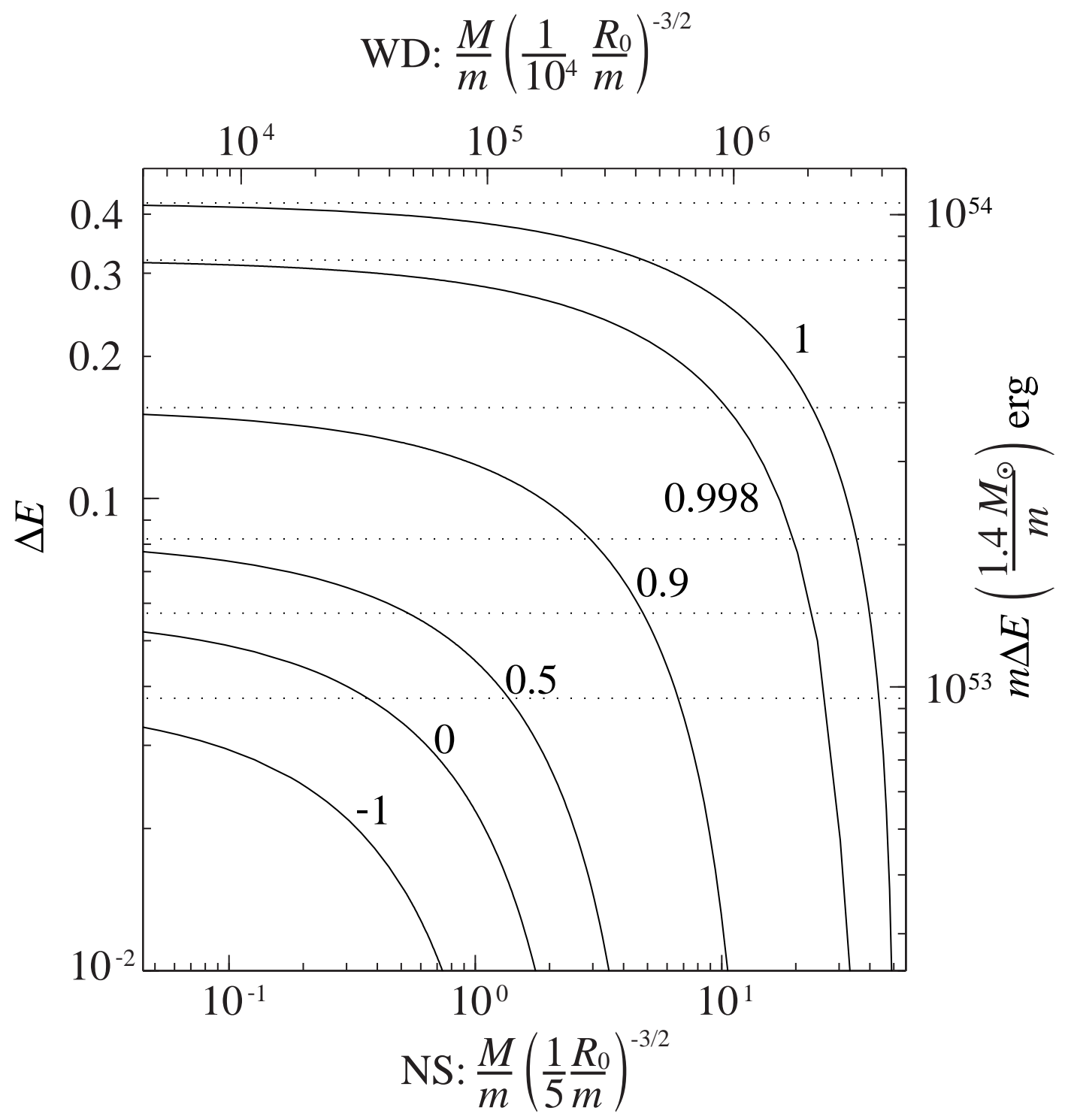

Fig. 8. - The maximum energy available (per unit mass of accreting matter), $\Delta E=E\left(r_{\text {tide }}\right)-$ $E\left(r_{\text {isco }}\right)$, in the accretion disk following the tidal disruption, is plotted against the "effective" mass ratio. The numerical value adjacent to each solid curve indicates the Kerr parameter $\hat{a}$. $(\hat{a}=$ 0.998 is the Thorne limit for Kerr BHs fed by accretion disks.) The dotted horizontal lines indicate the limiting energy for disruption at $\infty$ for each of the Kerr parameters. For BH-NS binaries, the bottom horizontal axis shows the mass ratio scaled for $R_{0} / m=5$ (Results for $M / m$ less than a few can only be viewed suggestively, and results below $M / m=1$ is not physically meaningful). For BH-WD binaries, the top horizontal axis shows the mass ratio scaled for $R_{0} / m=10^{4}$. The label on the right gives the total energy in ergs for $m=1.4 M_{\odot}$. Note that in this figure, $r_{\text {tide }}$ is calculated for $n=0$, but the dependence of $\Delta E$ on $n$ is weak. 